\title{
Assessment of Random and Blocked Practice Schedules on Motor Skills' Acquisition, Retention and Transfer Among Selected Senior High School Students
}

\author{
Srem-Sai Medina ${ }^{1}$, Hagan Jr John Elvis ${ }^{1}$, , Jatong Ahmed Baba ${ }^{3}$, Schack Thomas ${ }^{2}$ \\ ${ }^{1}$ Department of Health, Physical Education and Recreation, University of Cape Coast, Cape Coast, Ghana \\ ${ }^{2}$ Neurocognition and Action - Biomechanics- Research Group, Faculty of Psychology and Sport Sciences/CITEC, Bielefeld University, \\ Bielefeld, Germany \\ ${ }^{3}$ Department of Health, Physical Education, Recreation, and Sports, University of Education, Winneba, Ghana
}

Email address:

elvis.hagan@ucc.edu.gh (H. Jr J. Elvis)

${ }^{*}$ Corresponding author

\section{To cite this article:}

Srem-Sai Medina, Hagan Jr John Elvis, Jatong Ahmed Baba, Schack Thomas. Assessment of Random and Blocked Practice Schedules on Motor Skills' Acquisition, Retention and Transfer Among Selected Senior High School Students. American Journal of Sports Science. Vol. 7, No. 1, 2019, pp. 26-33. doi: 10.11648/j.ajss.20190701.15

Received: February 21, 2019; Accepted: April 2, 2019; Published: May 9, 2019

\begin{abstract}
The purpose of this study was to assess the influence of blocked and random practice schedules on acquisition, retention and transfer after teaching basketball skills among novice Senior High School students. Using a quasi-experimental design, a combination of simple random and purposive sampling procedures were used to select 60 novice participants who were equally assigned to a Blocked Practice Group (BPG [N=30]) and Random Practice Group (RPG [N=30]) respectively. After 9 sessions of teaching and learning of chest pass, overhead pass and sidearm pass, results for skill acquisition indicated that the BPG scored higher means than the RPG in all the three motor skills. For retention, improved performance for the RPG than the BPG in all 3 skills was noted. Similar results were obtained for the transfer phase. ANOVA test for retention produced significant mean differences between the three motor skills. However, the RPG recorded more superior values for between group analyses than the BPG. Independent sample t-test also revealed significant difference between random and blocked practices in terms of overall learned skills. Based on these findings, Physical education tutors and perhaps coaches should consider adopting blocked practice approach during isolated skill learning. Additionally, random practice scheduling should be used when the main objective is based on retention and transfer of knowledge regarding motor skills, especially during competitive milieu or other analogous related activities.
\end{abstract}

Keywords: Blocked, Random, Practice, Contextual Interference, Acquisition, Retention, Transfer

\section{Introduction}

Research to date has tried identifying significant determinants underlying the acquisition of motor skills to better understand the various processes that influence a learning process $[1,2]$. Of particular interest to researchers in motor learning is practice organization, considered as a powerful instructional approach generally seen as a significant factor responsible for the improvement in the ability to acquire, retain, perform and possibly transfer a motor skill [3, 4]. All things being equal, motor skill development is generally considered to be positively associated to the amount of practice [5]. Therefore, individuals who devote substantial amount of time through practice-related activities improve performance. Conversely, performers who spend little time on practice, usually show less improvements and suffer performance decline [6,7].

Importantly, the type of practice schedule appears to impact learners' assessments of their competency level [8]. According to Dail and partner [8], these assessments denote a learner's confidence which significantly account for his or her judgment on current proficiency level that determines how much more practice on a motor task is required. One of 
the most commonly studied variables is the impact of random and blocked practice, commonly referred to as "contextual interference" effect $[9,10]$. The extent to which a teacher or coach stresses on one type of practice approach over the other defines the magnitude of contextual interference offered within each practice schedule [9]. According Williams \& Hodge [2], a low contextual interference practice session may possibly involve practising one motor skill per session, or perhaps two separate motor skills (e.g., passing, shooting) in blocks of $20-30$ minutes each (i.e., blocked practice). Comparatively, a higher contextual interference would happen if varied skills (e.g., passing, dribbling, shooting) were utilized to a certain degree in a random manner during a practice session (i.e., random practice). During a random practice session, a learner by no means performs the same skill on consecutive trials. The impact shows that individuals or learners who practice in high levels of contextual interference (e.g., a random arrangement of trials on varied tasks) have sub-standard performance outcomes during skill acquisition but show superior retention and subsequent transfer of motor skills learnt compared to low levels of contextual interference (e.g., a blocked practice approach where all practice trials of one task are performed before another task [10]). Literature on contextual interference effect shows that blocked practice encourages a poor level of cognitive processing resulting in task difficulty, cf $[11,12]$. Blocked practice therefore leads to a kind of rote learning that allows for better performance during training sessions but less skill transfer to competitions and novel situations, as well as lower retention levels over time. One explanation for this is that, there are lower demands on active problem-solving and engagement during blocked practice than during random practice [13]. This effect is more pronounce when learning motor skills that are relatively simple in task difficulty [5].

The underlying principle is that although random practice has negative effects on performance during acquisition, it compensates learning by either reassuring the learner to carry out more defined and elaborate processing from one trial to the other (i.e., the elaboration hypothesis; see [10]) or through the forgetting and subsequent reconstruction of an action plan each time a skill is executed (i.e., the action plan reconstruction hypothesis; see [11]). Primarily, "short term pain for long term gain" seems to hold true for the random practice approach, while "Short term gain for long term pain" seems to be true for the blocked practice design [14]. Effectively, a random or high contextual interference practice schedule, although negative towards short-term performance, has been shown to be better for long-term retention and learning than blocked conditions. These research outcomes have been confirmed in diverse sports like basketball [15], badminton [16], and baseball [17].

Despite overwhelming research evidence favouring random practice because of its associated benefits to the learner, some researchers have argued that blocked practice is more appropriate for novice learners [15, 18-19]. For instance, Merbah and Meulemans [20] indicate that skill acquisition in novice subjects tends to be higher in blocked practice conditions while highly skilled subjects take advantage of random practice conditions in both retention and transfer. Along a task difficulty continuum (i.e., nominal and functional task difficulty), a beginner's performance outcome is expected to be high under conditions of very low nominal task difficulty. As the task becomes more difficult, the expected level of performance for the beginner drops rapidly. Performance then drops at a relatively low level of task difficulty. For the expert, only the most nominally difficult tasks would be expected to pose a problem. Therefore, if the task nominal difficulty increases, performance will decrease and the rate of decline in performance will be more rapid for the lower-skilled performer. Therefore, when the learner is relatively inexperienced, random practice may overload the system and its potential benefits could be disrupted [21].

To date, no single study has accounted for the impact of varied practice schedules across any population in Ghana. Additionally, despite researchers devoting considerable time to document practice profiles of elite performers, thus significantly improving knowledge on how to reach elite level [2, 22], there remains sparse information on which specific practice parameters could account for motor skill acquisition, retention and transfer among novices. Janelle and Hillman [23] highlighted the importance of conducting additional research that would determine the "what" and "how" of practice and how these indicators may influence instructional process. This current study therefore, considered two teaching methodological approaches - a high variability random practice and a low variability blocked practice to determine the extent to which these two practice schedules facilitate acquisition, retention and transfer of three taught novel basketball skills: chest pass (CP), overhead pass (OP), and sidearm pass (SP) during standard Senior High School Physical Education lessons. Specifically, we examined the blocked (BP) and random (RP) practice approaches in skill acquisition and determine which of the two methodological approaches to teaching novel motor skills in basketball would produce better retention. It was anticipated that learnt technical skills would be transferred successfully in natural field settings.

\section{Materials and Methods}

\subsection{Participants}

Sixty $(n=60)$ adolescent students, aged between 14-18 years from Presbyterian Senior High School in the Greater Accra Region of Ghana participated in this study. Participants were randomly assigned to two treatment groups with equal representation; the BPG $(n=30)$ and RPG $(n=$ 30) before completing a practice trial. All participants had self-reported normal or corrected-to-normal vision and were right-handed, with the exception of one left-handed participant in each group. The study was undertaken according to the university's ethical guidelines. No 
participant had prior experience with the task. All participants signed an informed consent form before participation in the study. The study design employed was a pretest - posttest quasi-experimental approach to determine the effect of practicing three basketball skills (chest pass, overhead pass and sidearm pass) on skill acquisition, transfer and retention phases.

\subsection{Task/Apparatus}

The main instruments used for data collection were three basketball passing skills tests and a wall with standard criteria measurements. The basketball passes employed for the study were the chest pass, overhead pass, and sidearm pass. These three passes were selected because they are basic basketball passes often preferred for the teaching of novice learners. For the measurements on the wall, 20 horizontal lines with $10 \mathrm{~cm}$ space between each two lines were drawn on a wall at a 4-meter distance from the participants (during the acquisition stage). The lines were scaled from -9 to +9 from top to bottom so that passing toward the space between the two uppermost lines and two lowermost lines would equal a score of -9 or +9 respectively.

\subsection{Procedure}

Permission was sought from the Headmaster of the school to allow for the selected students to be used as participants for the study. All participants were required to have their parent's consent for participation as well as their own willingness to participate in the study. Two qualified and experienced basketball coaches were invited to assist in training of the participants. A pre-training session with the coaches was conducted by one of the researchers on the test protocol. The coaches were initially briefed on the rational of the study and the procedures or methodological approaches to be adopted in their training. Before test the administration began, an introductory session was held, where one of the coaches provided explanations on the three different types of basketball passes (chest pass, overhead pass and sidearm pass) to the study participants. The scoring process was also elaborated by the one of the researchers. Although the three skills mentioned above had different structures and different motor skill programmes, the number of trials and distance from targets were equal for all the passes. In the experiment, participants stood at a 4-meter distance away from the wall and passed the ball toward the zero (0) point such that the ball did not hit the ground. A pre-test was conducted at the end of the introductory session to eliminate any learning effects and eliminate or control for some extraneous variables. The pre-test was done to ensure that all participants were almost equivalent at a beginner level in basketball. At the skill acquisition level, each participant in a group was made to perform 27 trials for each pass and a total of 81 trials during 9 sessions of practice ( 9 trials per session). The blocked practice group (BPG) performed 27 trials of the first pass (chest pass), 27 trials of the second pass (overhead pass) and 27 trials of the third pass (sidearm pass). The random practice group (RPG) performed their trials in no specific order. Not more than two consecutive trials could occur for anyone of the passes. Each group trained at different times with an assigned coach. Performances of participants during each of the nine practice sessions were recorded on assessment sheets and constantly scrutinized to be sure the correct practice approach with the test items had been implemented. A post-test on acquisition, retention and transfer of the three basketball passes was conducted to examine how students had learned. The acquisition test was conducted soon after the nine practice sessions whereas a retention test was done a day after the practice period with 4 trials for each pass in a mini blocked practice schedule $(2$ trials each for chest; overhead; sidearm passes continued until 12 trials were performed). For the transfer test, 4 trials of each pass at a 5-meter distance from the wall were performed by each participant two weeks after the practice sessions.

\subsection{Data Analysis}

Data analysis was done with different procedures. An initial data prescreening was conducted to check on the accuracy of the data and statistical assumptions. Second, descriptive statistics (i.e., mean scores and standard deviations) for each practice approach on the three skills being learned across acquisition, retention, and transfer phases were carried out. To examine the differences between the pre-test and post-test mean scores, a 2 (groups) by 3 (measurement periods) analysis of variance was used to evaluate the significant effect of these learnt skills. Third, an independent sample t-test was also used to test for significant differences after skills practice sessions between the random practice and blocked practice groups. All analyses were performed at a set alpha, $p<.05$.

\section{Results}

\subsection{Descriptive Statistics}

\subsubsection{Acquisition Phase}

The means and standard deviations scores of the two groups (RPG \& BPG) based on the skills taught (chest pass, overhead pass \& sidearm passes in Basketball) after an hour practice are presented in Table 1.

The chest pass showed an average score, 28.57 for the random practice group (RPG) whereas for blocked practice group (BPG), an average mean score of 33.73 was realized. For the overhead pass, the RPG had a mean score of 29.57 while the BPG recorded a mean score of 34.07. The mean scores for RPG and BPG were 28.97 and 33.87 for the sidearm pass respectively. 
Table 1. Means and Standard Deviation Scores of Participants' Performance in Basketball Skills after an Hour of Skills Practice.

\begin{tabular}{|c|c|c|c|c|c|}
\hline & & $\mathbf{N}$ & Mean & Std. Deviation & Std. Error \\
\hline \multirow{3}{*}{ Chest pass } & Random & 30 & 28.57 & 2.079 & .380 \\
\hline & Blocked & 30 & 33.73 & 1.081 & .197 \\
\hline & Total & 60 & 31.15 & 3.080 & .398 \\
\hline \multirow[t]{2}{*}{ Overhead pass } & Blocked & 30 & 34.07 & .785 & .143 \\
\hline & Total & 60 & 31.82 & 2.771 & .358 \\
\hline \multirow{2}{*}{ Side Arm Pass } & Random & 30 & 28.97 & 2.697 & .492 \\
\hline & Total & 60 & 31.42 & 3.196 & .413 \\
\hline
\end{tabular}

\subsubsection{Retention Phase}

Table 2 below indicates that mean score for chest pass was 33.17 for random practice, whereas the blocked practice revealed a lower score of 29.07. The sidearm pass was slightly higher in random practice (33.33) than in blocked practice (30.27) while in the overhead pass, the mean scores for random and blocked practices were 32.77 and 29.63 respectively. The results revealed that random practice after a day of skill training produced more favourable results than blocked practice. While results for random practice were higher than those for blocked practice after an hour of practice (Table 2), the results obtained after a day of skill training showed a decrease in the means of the blocked practice groups $(29.07,30.27$ and 29.63) as against that of random practice $(33.17,33.33$ and 32.77 ) which were improved for the chest, sidearm and overhead passes respectively.

Table 2. Means and Standard Deviation Scores of Participants' Performance in Basketball Skills after a Day of Skills Practice.

\begin{tabular}{llllll}
\hline & N & Mean & $\begin{array}{l}\text { Std. } \\
\text { Deviation }\end{array}$ & $\begin{array}{l}\text { Std. } \\
\text { Error }\end{array}$ \\
\hline \multirow{2}{*}{ Chest } & Random & 30 & 33.17 & 1.599 & .292 \\
pass & Blocked & 30 & 29.07 & 1.818 & .332 \\
& Total & 60 & 31.12 & 2.675 & .345 \\
Side Arm & Random & 30 & 33.33 & 1.213 & .221 \\
Pass & Blocked & 30 & 30.27 & 2.196 & .401 \\
& Total & 60 & 31.80 & 2.342 & .302 \\
Overhead & Random & 30 & 32.77 & 1.382 & .252 \\
pass & Blocked & 30 & 29.63 & 1.650 & .301 \\
& Total & 60 & 31.20 & 2.185 & .282 \\
\hline
\end{tabular}

\subsubsection{Transfer Phase}

From Table 3 below, the mean scores realized for the overhead pass were 34.23 and 28.00 for random and blocked practices respectively. However, in the sidearm pass, random practice produced a mean score of 34.17 while blocked practice recorded a mean score of 28.87. The mean scores for chest pass during the random and blocked practices were 33.83 and 27.87 respectively. After two weeks practice sessions, the mean scores for RPG improved $(34.23,34.17$ and 33.83) while that of BPG declined (28.0, 28.87 and 27.87).

Table 3. Means and Standard Deviation Scores of Participants' Performance in Basketball Skills after 2-Weeks of Skills Practice.

\begin{tabular}{llllll}
\hline & & N & Mean & $\begin{array}{l}\text { Std. } \\
\text { Deviation }\end{array}$ & $\begin{array}{l}\text { Std. } \\
\text { Error }\end{array}$ \\
\hline Overhead & Random & 30 & 34.23 & 1.040 & .190 \\
pass & Blocked & 30 & 28.00 & 1.983 & .362 \\
& Total & 60 & 31.12 & 3.513 & .454 \\
Side Arm & Random & 30 & 34.17 & 1.053 & .192 \\
Pass & Blocked & 30 & 28.87 & 2.662 & .486 \\
& Total & 60 & 31.52 & 3.342 & .431 \\
Chest & Random & 30 & 33.83 & 1.440 & .263 \\
pass & Blocked & 30 & 27.87 & 2.897 & .529 \\
& Total & 60 & 30.85 & 3.768 & .486 \\
\hline
\end{tabular}

\subsection{ANOVA Results on Retention and Transfer of Motor Skills}

To determine whether significant differences exist between the teaching approaches used for the learned basketball skills, ANOVA analysis was computed. The results after a day of practice revealed significant differences among the three skills: chest pass, $\mathrm{F}_{(1,58)}=86.01, \mathrm{p}<.05$; sidearm pass, $\mathrm{F}_{(1,58)}$ $=44.82, \mathrm{p}<.05$; and overhead pass, $\mathrm{F}_{(1,58)}=63.58, \mathrm{p}<.05 \mathrm{a}$ day after practice. The $F$ values show a significant difference in the means of skill performances on retention of the 3 basketball skills between the RPG and BPG.

Table 4. Results of ANOVA Test for Participants'Skills Performances in the Random and Blocked Groups after a Day of Practice.

\begin{tabular}{|c|c|c|c|c|c|c|}
\hline ANOVA & & Sum of Squares & df & Mean Square & $\mathbf{F}$ & Sig. Value \\
\hline \multirow{3}{*}{ Chest pass } & Between Groups & 252.150 & 1 & 252.150 & 86.011 & $.000^{*}$ \\
\hline & Within Groups & 170.033 & 58 & 2.932 & & \\
\hline & Total & 422.183 & 59 & & & \\
\hline \multirow{3}{*}{ Side Arm Pass } & Between Groups & 141.067 & 1 & 141.067 & 44.824 & $.000^{*}$ \\
\hline & Within Groups & 182.533 & 58 & 3.147 & & \\
\hline & Total & 323.600 & 59 & & & \\
\hline \multirow{2}{*}{ Overhead pass } & Between Groups & 147.267 & 1 & 147.267 & 63.584 & $.000^{*}$ \\
\hline & Total & 281.600 & 59 & & & \\
\hline
\end{tabular}

${ }^{*} \mathrm{P}<0.05=$ significant results 
The results after 2 weeks of practice also showed significant differences among the three skills: chest pass, $\mathrm{F}_{(1}$, ${ }_{58)}=232.54, \mathrm{p}<.05$; sidearm pass, $\mathrm{F}_{(1,58)}=102.84, \mathrm{p}<.05$; and overhead pass, $\mathrm{F}_{(1,58)}=102.01, \mathrm{p}<.05$ (see Table 5) respectively.

Independent-Samples T-Test on 2 Measurements (Retention and Transfer Phases)

An independent-samples t-test was conducted to compare the mean scores the groups (RPG and BPG) in the retention and transfer of learned skills. After a day of practice, there was significant difference in mean scores for RPG $(M=33.07$, $S D=1.14)$ and BPG $([M=29.70, S D=1.159] ; t(58)=11.38$, $p<.05)$. Similarly, there was also a significant difference in mean scores for RPG $(M=34.03, S D=.77)$ and BPG $([M=28.23, S D=1.63] ; t(58)=17.61, p<.05)$ after 2 weeks of practice.

Table 5. Results of ANOVA Test for Participants'Skills Performances in the Random and Blocked Groups after 2-Weeks of Practice.

\begin{tabular}{|c|c|c|c|c|c|c|}
\hline ANOVA & & Sumof Squares & df & Mean Square & $\mathbf{F}$ & Sig. Value \\
\hline \multirow{3}{*}{ Overhead pass } & Between Groups & 582.817 & 1 & 582.817 & 232.539 & $.000 *$ \\
\hline & Within Groups & 145.367 & 58 & 2.506 & & \\
\hline & Total & 728.183 & 59 & & & \\
\hline \multirow{3}{*}{ Side Arm Pass } & Between Groups & 421.350 & 1 & 421.350 & 102.840 & $.000 *$ \\
\hline & Within Groups & 237.633 & 58 & 4.097 & & \\
\hline & Total & 658.983 & 59 & & & \\
\hline \multirow{2}{*}{ Chest pass } & Between Groups & 534.017 & 1 & 534.017 & 102.008 & $.000 *$ \\
\hline & Total & 837.650 & 59 & & & \\
\hline
\end{tabular}

$* \mathrm{P}<0.05$ = significant result

\section{Discussion}

Considerable research evidence from laboratory findings suggest that contextual influence support skill acquisition, retention and transfer of motor skills [24, 25]. However, the application and generalizability on these laboratory findings on field based intact teaching and learning of motor skills ought to be verified through additional field-based research. The central focus of this present study was to analyze which type of practice method (random or blocked) would influence acquisition, retention and transfer after teaching three different basketball skills (chest pass, overhead pass, and sidearm pass) among novices, aged between 14-18 years during standard Senior High School Physical Education lessons.

Findings revealed that after an hour of skill practice, both blocked and random practice approaches influenced the acquisition of the three basketball skills (chest pass, overhead pass and sidearm pass). Even though participants fell in the same continuum of knowledge gained from their learning, the blocked practice method produced a higher mean score than the random practice approach. According to the Schmidt's Schema theory, successful performance of a skill depends on the amount of variability of practice [26]. The variability of environmental features experienced by participants during the practice of skills may influence subsequent performance and that beginner learners will learn more effectively if they begin with blocked practice [27]. Within the blocked practice schedules, subjects are faced with few challenges in the early stages of practice, but compensates for these challenges with number of trials to achieve optimal performance in acquisition of skills. Other studies, [28, 29] further confirm that relative to random practice of several movements at a time, practice of one movement by novice learners during a trial may account for superior performance in skill acquisition because of low interference conditions.

Guadagnoli and Lee [5] suggested that performance level can also be linked with task difficulty for subjects with different levels of expertise and identifies difficulty along two dimensions: nominal task difficulty and functional task difficulty. Using a task of a given level of nominal difficulty, an individual at any skill level is likely to perform at a predictable level. For a beginner, performance outcome is expected to be high only under conditions of very low nominal task difficulty. However, the results of the current study do not agree with several other findings [30, 31] which found inconsistent results using the random and blocked practice methods.

Similar to previous studies [4, 32], the random practice approach produced higher performance outcomes (i.e., mean values) than the blocked practice schedule in all the three skills tested for retention and transfer (after a day and 2 weeks of practice), thus showing support for the retroactive interference theory [33]. The blocked practice approach recorded lower mean scores on performance at the initial stages of skill learning in the current study. According to the retroactive interference theory, later learned patterns in blocked practice schedules tend to act backwards to attenuate the memory strength of earlier learned skill patterns. However, in random practice, the individuals do not finish a skill before starting the next skill and are not subjected to the disadvantages of retroactive interference. Additionally, in most random practice schedules, players do not practice the same skill over consecutive trials. Despite research suggesting that random practice has negative effects on performance during acquisition, it aids learning either by boosting performers toward using more elaborate and distinctive processing from one trial to the other (i.e., the elaboration hypothesis; see [10]) or through forgetfulness and the later reconstruction of an action plan any time a skill is 
executed (i.e., the action plan reconstruction hypothesis; see [11]). Further, the random practice approach may widen the learning workspace; thereby facilitating more prospects for players to use discovery learning to identify diverse resolutions to movement challenges encountered [34].

From the ANOVA and t test results, the random practice schedule produced progressively significant results commonly seen with retention and transfer of learned skills. Random practice schedules therefore improve learning because processing is more elaborate, distinct and meaningful to the learner and information processing is more elaborate and/ or extensive compared to individuals under blocked conditions who engage in intra-task processing through repetitive practice of the same skill. The inter-task processing mode helps to identify the similarities and differences between the motor tasks being learned by between-task analyses. This mode is one that elaborates the existing motor (task) representation and increases the depth of the reclamation routes available to the performer to access task relevant cues (i.e., information) to effectively perform the task upon acquisition [12, 35]. These authors [12, 35] reiterated that under random practice, there is usually a persistent exchange of information that is located within the working memory in order for performers to calibrate and execute a correct response. The elaboration hypothesis alludes to the superior retention and subsequent transfer of performance after undergoing a high contextual interference practice condition regarding the usage of more extensive intra-item processing [12] and, more significantly, the use of inter-item processing strategies [36, 37]. Comparatively, participants in a low contextual interference practice condition (i.e., blocked practice) are restricted to the usage of only intra-task processing, as this medium of schedule warrants the learner to concentrate on just one task at a time during practice. In essence, it is vital to acknowledge that trial-to-trial repetition of a task in blocked practice primarily decreases the chance of the cues specific to that task. These cues may be forgotten at the beginning of each subsequent trial for that same task. Hence, minimal reconstruction of movement planning is necessary because the essential information already exist in the working memory as a prerequisite for the necessary basic action concepts that ought to be hierarchically structured to be retrieved during performance of that motor task [11, 38-39].

\section{Practical Implications for Teachers and Coaches}

The take-home message is that to promote motor skills via learning, teachers and coaches ought to minimize repetitive blocked practice by offering diverse motor skills within the same practice schedule. Other studies $[2,40]$ have shown that the gains from random practice seem to be enriched when motor skills appear to vary (e.g., dribbling with a chest pass may elicit more effect than the chest pass, overhead pass and sidearm pass in basketball) as captured in the current study
[40]). However, some empirical evidence, including this study suggest that blocked practice have some benefits during the acquisition of motor skills over random practice $[12,18$, 35].

According to Williams and Hodges [2], while specific blocked practice is better for motor performance, random practice is more effective for progressive motor skill learning. Williams and co-worker reiterated the arduous task that may confront coaches and teachers on when to present variation within the random practice schedules. The challenge would be the prospect of maintaining constructive performance effects on one hand, so that learners would persist to practice, while boosting effective learning on the other hand [41]. Given that teachers and coaches have the penchant toward introducing new variables as the learners' progress with the random practice condition, the level of progression may happen at a slower rate than the ideal situation. Teachers and coaches ought to provide learners with a time lag for performance improvements before moving on to complex motor skill practices [2, 40]. Teachers and coaches should attempt to appraise motor skill learning over prolonged periods (e.g., multiple practice sessions) rather than one snap shot assessment and reflect on whether learners would accrue any benefit from an earlier practice towards more random practice schedules. Coker [42] suggested that the effectiveness of a training schedule should not be measured by the speed of acquisition or the level of performance reached at the end of practice opportunities by learners but rather, by the learners' performance in realworld settings (i.e., novel and challenging situations), that is the reason for their training.

Summarily, the way novices practice motor skills relates to long-term retention and subsequent transfer. Several studies done on these two skill training approaches have shown that random practice is significantly better for long-term skill retention and application than the block practice approach. Arguably, the blocked practice approach is marked by low levels of cognitive interference whereas random practice is noted for its high levels of cognitive interference. Primarily, this means that random practice setups challenge the learner's cognitive and motor systems to deal with the interference of each task on the next activity; an element that keeps the performer alert and allows for greater retention and skill transfer. Further evidence from this study suggest that repetitive blocked practice facilitates rote learning that allows for better performance during training sessions but less skill transfer during competitions and novel situations, as well as lower retention levels over time. The demands on active problem-solving and engagement during blocked practice are lower than during random practice. Basically, during random practice, learners are forced to work through various skills presented randomly in a single training session, thus making adaptation of the cognitive system in order to execute appropriate motor patterns, upon demand. This would require an identification of similarities and differences among tasks before designating which motor pattern to calibrate and apply. 


\section{Conclusions}

Our primary findings in the present study are consistent with those of contextual interference literature on the effects of types of practice (i.e., block versus random) on learning motor skills. Even though the random practice schedules were less effective during the acquisition phase in the current study, they were better than the blocked practice conditions on retention test and subsequent transfer. These variations are remarkable given the ecological validity of the current study; that is, real-field based learning experiences. Relatively, it is more effective to practice under random conditions than under blocked conditions based on available scientific evidence. The random practice manipulation produces performance decline in acquisition yet, fosters performance enhancement at retention and contributes to the ability to perform in diverse context (transfer). Relative amount of motor skill learned should be assessed by performance on retention tests, and that performance levels in acquisition could be equivocal, with respect to the amount of skill learned. In most educational and training settings, learning and retention are seen as two different learning constructs. "Learning" refers to that set of processes occurring during the actual practice of motor tasks, as measured by performance indicators taken at that time, whereas "retention" involves the set of processes that manifest after practice is completed, during some retention interval, and prior to a retention test. Because learning and retention are thought to be different phenomena, they tend to be studied with separate methods by different scientists. Instead of seeing motor skill learning (i.e., acquisition) and retention as distinct phenomena, the effectiveness of motor skill acquisition is shown by the level of retention produced which invariably facilitates future use of the motor skills learned in different circumstances.

\section{Authors' Declaration}

The authors declare that they have no competing interests

\section{Acknowledgements}

Sincere thanks to the Headmaster, Presbyterian Senior High School in the Greater Accra Region of Ghana for supporting this research. Gratitude also goes to the staff and students for helping with the data collection. The School of Graduate Studies, University of Education, Winneba- Ghana and the Neurocognition and Action-Biomechanics Research Group, Bielefeld University, Germany deserve acknowledgement for providing financial support for this research.

\section{Authors' Contributions}

MSS conceived the study, designed and performed the analysis, and the write up on the data. JEH and JB produced the first draft of the manuscript. MSS, JEH, JB and TS revised and proof read the manuscript for its intellectual content and approved for the final version for publication.

\section{References}

[1] Keetch KM, Lee TD. The effect of self-regulated and experimenter-imposed practice schedules on motor learning for tasks of varying difficulty. Research quarterly for exercise and sport. 2007; 78(5): 476-86.

[2] Williams AM, Hodges NJ. Practice, instruction and skill acquisition in soccer: Challenging tradition. Journal of sports sciences. 2005; 23(6): 637-50. 10.1080/02640410400021328

[3] Magill R. A. Motor learning: Concepts and applications (6th ed.). Madison, WI: Brown \& Benchmark. 2001.

[4] Schmidt, RA, Lee, TD. Motor learning and control: A behavioral emphasis $\left(4^{\text {th }}\right.$ edn.). Champaign, IL: Human Kinetics. 2005.

[5] Guadagnoli MA, Lee TD. Challenge point: a framework for conceptualizing the effects of various practice conditions in motor learning. Journal of motor behavior. 2004; 36(2): 21224. 10.3200/JMBR.36.2.212-224

[6] Magill, RA. Motor learning and control: Concepts and applications $\left(9^{\text {th }}\right.$ edn. $)$. New York: McGraw- Hill. 2011.

[7] Ward, P, Williams, AM. Perceptual and cognitive skill development in soccer: The multidimensional nature of expert performance. Journal of Sport and Exercise Psychology. 2003; 25: $93-111$.

[8] Dail TK, Christina RW. Distribution of practice and metacognition in learning and long-term retention of a discrete motor task. Research quarterly for exercise and sport. 2004; 75(2): 148-55.

[9] Broadbent, DP, Causer, J, Ford, PR, Williams, AM. Contextual interference effect in perceptual-cognitive skills training. Medicine \& Science in Sports \& Exercise. 2015; 47: 1243-1250.

[10] Shea JB, Morgan RL. Contextual interference effects on the acquisition, retention, and transfer of a motor skill. Journal of Experimental psychology: Human Learning and memory. 1979; 5(2): 179.

[11] Lee TD, Magill RA. Can forgetting facilitate skill acquisition? In D. Goodman, R. B. Wilberg, \& I. M. Franks (Eds.), Differing perspectives in motor learning, memory, and control (pp. 3-22). Amsterdam: Elsevier. 1985.

[12] Boyce, BA, Coker, CA, Bunker, LK. Implications for variability of practice from pedagogy and motor learning perspectives: Finding common ground. Quest. 2006; 58: 330-343.

[13] Allison, B. Blocked vs. random practice: Shake things up in your training and in your life. 2013. http://www.goldigest.com/magazine/2011-03/kaspriskefitness-column.

[14] Otte, B, Zanic V. Blocked versus random practice with drills for hurdlers. 2008, http//speedendurance.com./rss.

[15] Landin D, Hebert EP. A comparison of three practice schedules along the contextual interference continuum. Research quarterly for exercise and sport. 1997; 68(4): 35761. 
[16] Goode S, Magill RA. Contextual interference effects in learning three badminton serves. Research Quarterly for Exercise and Sport. 1986; 57(4):308-14.

[17] Hall, KG, Domingues, DA, Cavazos, R. Contextual interference effects with skilled baseball players. Perceptual and Motor Skills. 1994; 78: 835-841.

[18] Ward, P, Hodges, NJ, Williams, AM, Starkes, JL. Deliberate practice and expert performance: Defining the path to excellence. In A. M. Williams and N. J. Hodges (Eds.), Skill acquisition in sport: Research, theory and practice (pp. $231-$ 258). London: Routledge. 2004.

[19] Pauwels, L, Swinnen, SP, Beets, IAM. Contextual interference in complex bimanual skill learning leads to better skill persistence. PlosOne. 2014; 9(6): e100906.

[20] Merbah, S, Meulemans, T. Learning a motor skill: Effects of blocked versus random practice - A review. 2011. http://dx.doi.org/10.5334/pb-51-1-15.

[21] Guadagnoli, MA. Challenge point: A framework for conceptualizing the effects of various practice conditions in motor learning. Journal of Motor Behavior. 2004; 36: 212224.

[22] Ward, P, Hodges, NJ, Williams, AM, Starkes, JL. Deliberate practice and expert performance: Defining the path to excellence. In A. M. Williams and N. J. Hodges (Eds.), Skill acquisition in sport: Research, theory and practice (pp. $231-$ 258). London: Routledge. 2004.

[23] Janelle, CM, Hillman, CH. Expert performance in sport: Current perspectives and critical issues. In J. L. Starkes \& K. A. Ericsson (Eds.), Expert performance in sports: Advances in research on sport expertise (pp. $19-48$ ). Champaign, IL: Human Kinetics. 2003.

[24] Shea, CH, Kohl, RM. Specificity and variability of practice. Research Quarterly for Exercise and Sport. 1990; 62: 187-195.

[25] Shea, CH, Kohl, RM. Composition of practice: Influence of the retention of motor skills. Research Quarterly for Exercise and Sport. 1991; 62: 187-195.

[26] Schmidt RA. Motor schema theory after 27 years: Reflections and implications for a new theory. Research Quarterly for Exercise and Sport. 2003 Dec 1; 74(4): 366-75.

[27] Magill, RA, Anderson, D. Motor learning and control: Concepts and applications. New York, NY: McGraw Hill. 2014.

[28] Heitman, RJ, Pugh, SF, Kovaleski, JE, Norell, PM, Vicory, JR. Effects of specific versus variable practise on the retention and transfer of a continuous motor skill. Perceptual and Motor Skills. 2005; 100: 1107-1113.
[29] Guadagnoli, MA, Holcomb, WR, Weber, T. The relationship between contextual interference effects and performer expertise on the learning of a putting task. Journal of Human Movement Studies. 1999; 37: 19-36.

[30] Maslovat, D, Chua, R, Lee, TD. Franks, IM. Contextual interference: Single task versus multi-task learning. Journal of Motor Control. 2004; 8: 213-233.

[31] Zetou, E, Michalopoulou, M, Giazitzi, K. Kioumourtzoglou, E. Contextual interference effects in learning volleyball skills. Perceptual and Motor Skills. 2007; 104: 995-100.

[32] Porter, JM, Landin, D, Hebert, EP, Baum, B. The effects of three levels of contextual interference on performance outcomes and movement patterns in golf skills. International Journal of Sports Science and Coaching. 2007; 2: 243-255.

[33] Barreiros, J, Figueiredo, T, Godinho, M. The contextual interference effect in applied settings. European Physical Education Review. 2007; 3: 195-208.

[34] Handford, C, Davids, K, Bennett, S, Button, C. Skill acquisition in sport: Some applications of an evolving practice ecology. Journal of Sports Sciences. 1997; 15: 621 - 640.

[35] Lee, T, Wishart, LR. Motor learning conundrums (and possible solutions). Quest. 2005; 57: 67-78.

[36] Magnuson, CE, Wright, DL. Random practice can facilitate the learning of tasks that have different relative time structures. Research Quarterly for Exercise and Sport. 2004; 75:197-202.

[37] Porter, JM, Magill, RA. Systematically increasing contextual interference is beneficial for learning sport skills. Journal of Sports Sciences. 2010; 12: 1277-1285.

[38] Frank, C, Land, WM, Schack, T. Development of mental representations during learning of a complex action. Psychology of Sport and Exercise. 2013; 14: 353e361. http://dx.doi.org/10.1016/j.psychsport.2012.12.001.

[39] Schack, T, Mechsner, F. Representation of motor skills in human long-term memory. Neuroscience Letters. 2006; 391: 77-81. http://dx.doi.org/10.1016/j.neulet.2005.10.009.

[40] Brady, F. The contextual interference effect and sport skills. Perceptual and Motor Skills. 2008; 106: 461-472.

[41] Simon, DA, Bjork, RA. Metacogntion in motor learning. Journal of Experimental Psychology: Learning, Memory and Cognition. 2001; 27: 907-912.

[42] Coker, CA. Motor learning. New York, NY: McGraw-Hill Higher Education. 2003. 